\title{
Identification of DNA-Binding Proteins Using Support Vector Machine with Sequence Information
}

\author{
Xin Ma, ${ }^{1}$ Jiansheng $\mathrm{Wu},{ }^{2}$ and Xiaoyun $\mathrm{Xue}^{3}$ \\ ${ }^{1}$ Golden Audit College, Nanjing Audit University, Nanjing 210029, China \\ ${ }^{2}$ School of Geography and Biological Information, Nanjing University of Posts and Telecommunications, Nanjing 210046, China \\ ${ }^{3}$ Graduate School of Chinese Academy of Agricultural Sciences, Beijing 100081, China
}

Correspondence should be addressed to Xin Ma; maxin@nau.edu.cn

Received 13 May 2013; Accepted 19 August 2013

Academic Editor: Nestor V. Torres

Copyright (C) 2013 Xin Ma et al. This is an open access article distributed under the Creative Commons Attribution License, which permits unrestricted use, distribution, and reproduction in any medium, provided the original work is properly cited.

\begin{abstract}
DNA-binding proteins are fundamentally important in understanding cellular processes. Thus, the identification of DNA-binding proteins has the particularly important practical application in various fields, such as drug design. We have proposed a novel approach method for predicting DNA-binding proteins using only sequence information. The prediction model developed in this study is constructed by support vector machine-sequential minimal optimization (SVM-SMO) algorithm in conjunction with a hybrid feature. The hybrid feature is incorporating evolutionary information feature, physicochemical property feature, and two novel attributes. These two attributes use DNA-binding residues and nonbinding residues in a query protein to obtain DNA-binding propensity and nonbinding propensity. The results demonstrate that our SVM-SMO model achieves 0.67 Matthew's correlation coefficient (MCC) and $89.6 \%$ overall accuracy with $88.4 \%$ sensitivity and $90.8 \%$ specificity, respectively. Performance comparisons on various features indicate that two novel attributes contribute to the performance improvement. In addition, our SVM-SMO model achieves the best performance than state-of-the-art methods on independent test dataset.
\end{abstract}

\section{Introduction}

DNA-protein interaction has diverse functions in the cell, and it plays an important role in a variety of biological processes, such as gene regulation, DNA replication, and repair. Identification of DNA-binding proteins is the theoretical basis on many commonly used medicinal techniques. For instance, it is considered as selecting activators and inhibitors in rational drug design [1-3]. It also plays an essential role in discovering potential therapeutics for genetic diseases and proteome function annotation. Therefore, recognition of DNA-binding proteins becomes one of the most important questions in the annotation of protein functions.

In recent years, DNA-binding proteins can be annotated by several experimental techniques such as filter binding assays, X-ray crystallography, and NMR. However, experimental approaches to identify DNA-binding proteins remain time-consuming and expensive. Hence, the computational prediction of DNA-binding proteins is important. Most studies on computational prediction of DNA-binding proteins were based on structures of a query proteins [4-9]. But the problem of consuming time and money, arisen by procuring structure of protein, exist still yet. Therefore, it is important to develop computational methods for identifying DNAbinding proteins directly from amino acid sequence instead of structure information.

Machine learning technique is an effective tool which is widely used to distinguish DNA-binding proteins from nonbinding ones. Cai and Lin developed support vector machine (SVM) and the pseudoamino acid composition, a collection of nonlinear features extractable from protein sequence, to construct DNA-binding proteins prediction [10]. Yu et al. proposed the binary classifications for rRNA-, RNA-, and DNA-binding proteins using SVM and sequence features associated physicochemical properties [11]. A web-server DNAbinder (http://www.imtech.res.in/raghava/dnabinder/) has been developed for identifying DNA-binding proteins and domains from query amino acid sequences. It was constructed by SVM using amino acid composition and PSSM profiles [12]. Shao et al. constructed two classifiers to 
differentiate DNA/RNA-binding proteins from nonnucleicacid-binding proteins by using SVM and a conjoint triad feature which extract information directly from amino acids sequence of protein [13]. Patel et al. used an artificial neural network to identify DNA-binding proteins using a set of 62 sequence features [14]. Kumar et al. reported a random forest method, DNA-Prot, to identify DNA-binding proteins from protein sequence [15]. Lin et al. proposed a new predictor, called iDNA-Prot, for predicting uncharacterized proteins as DNA-binding proteins or non-DNA-binding proteins based on their amino acid sequences information alone [16].

In this study, we attempt to predict DNA-binding proteins directly from amino acid sequences. We propose a novel method for predicting DNA-binding proteins using a support vector machine-sequential minimal optimization (SVM-SMO) algorithm in conjunction with a hybrid feature. The hybrid feature is incorporating evolutionary information feature, physicochemical feature, and two novel attributes which represented DNA-binding propensity and nonbinding propensity. Those novel attributes were constructed by DNAbinding residues and nonbinding residues predicted by our previous work DNABR [17], respectively. Our model achieves 0.67 Matthew's correlation coefficient (MCC) and 89.6\% overall accuracy with $88.4 \%$ sensitivity and $90.8 \%$ specificity, respectively by 5 -fold cross-validation. In addition, the results demonstrate that the two novel attributes we propose in the research are discriminative to distinguish between DNAbinding proteins from nonbinding proteins.

\section{Materials and Methods}

2.1. Data. We collected DNA-binding proteins and nonbinding proteins from release "2013_02" of UniProtKB/Swiss-Prot database (http://www.uniprot.org/) [18]. To make sure of the reliability of data, we only selected manually annotated and reviewed proteins.

"DNA binding" was used as a keyword to search the UniProtKB/Swiss-Prot database. Then 29866 DNA-binding proteins were retrieved and designated as rough "Positive" dataset.

A "Contrast" dataset was obtained by the similar procedure which was proposed by Cai and Lin [10]. 158121 proteins in "Contrast" dataset were retrieved from UniProtKB/SwissProt database by searching with a list of keywords which possibly imply RNA/DNA-binding functionality using the "or" logic.

Then the proteins in "contrast" dataset were removed from UniProtKB/Swiss-Prot database, and 158121 proteins were obtained to form rough "Negative" dataset.

As indicated by previous research $[13,19]$, the protein sequences with the length range from 50 to 6000 amino acids are retained. Proteins including irregular amino acid characters such as " $x$ " and " $z$ " were removed. Moreover, the redundancy among the sequences in "positive" and "negative" datasets was removed by using BLAST package available from NCBI with a threshold of $40 \%$ identification. The longest amino acid sequence within each cluster was retained for reaching nonredundant dataset. Finally, 6653 and 60548 proteins were produced in nonredundant "Positive" dataset and
TABLE 1: The distribution of proteins in main dataset, training dataset, and independent test dataset.

\begin{tabular}{lccc}
\hline Dataset & $\begin{array}{c}\text { Number of } \\
\text { binding proteins }\end{array}$ & $\begin{array}{c}\text { Number of } \\
\text { nonbinding } \\
\text { proteins }\end{array}$ & $\begin{array}{c}\text { Total number } \\
\text { of proteins }\end{array}$ \\
\hline Main dataset & 6653 & 6653 & $\mathbf{1 3 3 0 6}$ \\
\hline $\begin{array}{l}\text { Training dataset } \\
\text { (TrD_10642) }\end{array}$ & 5321 & 5321 & $\mathbf{1 0 6 4 2}$ \\
$\begin{array}{l}\text { Independent test } \\
\text { dataset (TeD_2664) }\end{array}$ & 1332 & 1332 & $\mathbf{2 6 6 4}$ \\
\hline
\end{tabular}

"Negative" dataset, respectively. To deal with the imbalance problem between positive data and negative data, we created a "Negative subset" dataset by randomly selecting from "Negative" dataset which has the equal size to the "Positive" dataset. Therefore 13306 proteins contained in "Positive" dataset and "Negative subset" dataset consisted of the main dataset.

To evaluate the performance of our method against previous works $[15,16]$, an independent test dataset was used. 1332 DNA-binding proteins from "Positive" dataset and 1332 nonbinding proteins from "Negative subset" dataset were randomly selected to build independent test dataset. We made sure that the proteins in test dataset were not used in previous works $[15,16]$. Those remaining proteins in "Positive" dataset and "Negative subset" dataset were designated as the training dataset. Therefore, the training dataset (TrD_10642) obtained 10642 proteins and test dataset (TeD_2664) obtained 2664 proteins (more details can be seen from Table 1 and see Supplementary Material available online at http://dx.doi.org/10.1155/2013/524502).

\subsection{Feature Vector}

2.2.1. Binding Propensity and Nonbinding Propensity (BP and NBP). It is well known that DNA-binding residues should exist in DNA-binding protein and tend to appear on the surface of DNA-binding proteins. DNA-binding proteins have much more binding residues than nonbinding proteins and tend to gather together spatially. Therefore, these two characters of DNA-binding residues would be applied to identify binding proteins. We already had built a DNA-binding residue prediction model DNABR [17] (http://www.cbi.seu.edu.cn/DNABR/). The performance comparisons with other approaches showed that DNABR has an excellent prediction performance for detecting binding residues in putative DNA-binding protein. Consequently, we proposed binding propensity measures and nonbinding propensity measures which were made based on the prediction results of DNA-binding residues and nonbinding residues, respectively.

According to two characters of DNA-binding residues mentioned above, two binding propensity measures were defined for as follows:

$$
\mathrm{BP}(1)=\frac{\sum_{i=1}^{n} \mathrm{RI}(i)}{10 N},
$$


where $N$ is the number of amino acids in this protein, $n$ is the number of DNA-binding residues, and $\mathrm{RI}(i)$ is the predicted reliability index of DNA-binding residue $i$ obtained from DNABR. The reliability index is a positive integer range from 0 to 10 :

$$
\mathrm{BP}(2)=\frac{\sum_{i=1}^{N-1} 2^{-i+1} \sum_{k=1}^{n(i)} \overline{\mathrm{RI}}(k)}{10(N-1)},
$$

where $N$ is the number of amino acids in this protein, $n(i)$ is the number of two DNA-binding residues with the distance $i$, and $\overline{\mathrm{RI}}(k)$ is the mean of reliability index for DNA-binding residue $k$ and binding residue $k+i$.

For a query protein, $\mathrm{BP}(1)$ and $\mathrm{BP}(2)$ describe the information of the appearance and correlation of DNA-binding residues in the amino acid sequence, respectively. Due to the usage of predicted DNA-binding residue in this paper, reliability index is applied in $\mathrm{BP}(1)$ and $\mathrm{BP}(2)$ formula. $\mathrm{BP}(1)$ represents the frequency of DNA-binding residues. $\mathrm{BP}(2)$ represents the relevance of the two DNA-binding residues with different gaps from 1 to $N-1$ amino acids. The $\mathrm{BP}(2)$ formula takes into account the fact that the correlation value between two DNA-binding residues is smaller when the distance $k$ is larger.

Meanwhile, nonbinding proteins have two similar characters on nonbinding residues. Therefore, the definition of $\mathrm{NBP}(1)$ and $\mathrm{NBP}(2)$ is similar to that of $\mathrm{BP}(1)$ and $\mathrm{BP}(2)$ :

$$
\operatorname{NBP}(1)=\frac{\sum_{i=1}^{t} \mathrm{RI}(i)}{10 N},
$$

where $N$ is the number of amino acids in this protein, $t$ is the number of nonbinding residues, and $\mathrm{RI}(i)$ is the reliability index of prediction on nonbinding residue $i$ by DNABR:

$$
\operatorname{NBP}(2)=\frac{\sum_{i=1}^{N-1} 2^{-i+1} \sum_{k=1}^{t(i)} \overline{\mathrm{RI}}(k)}{10(N-1)},
$$

where $N$ is the number of amino acids in this protein, $t(i)$ is the number of two nonbinding residues with the distance $i$, and $\overline{\mathrm{RI}}(k)$ is the mean of reliability index for nonbinding residue $k$ and nonbinding residue $k+i$.

The vector size for BP features and NBP feature is 4dimensional.

2.2.2. Physicochemical Property (PP). Physicochemical property feature was usually used in the prediction of DNA/RNAbinding protein $[5,10,11,19,20]$, identification of proteinprotein interaction [21], protein fold recognition [22], and protein family classification [23]. This feature was constructed from amino acid composition and six biological properties of each amino acid including hydrophobicity, polarity, polarizability, secondary structure, solvent accessibility, and normalized Van der Waals volume.

The global composition of each physicochemical property was described by three descriptors, composition index, transition index, and distribution index. Composition index is the percent of amino acid of a particular property. Transition index is the percent frequency of which amino acid of a particular property is followed by amino acid of a different property. Distribution index measures the percent of length of a query protein within which the first $25 \%, 50 \%, 75 \%$, and $100 \%$ of the amino acid of a particular property are located respectively. Detail information of physicochemical property feature can be found in previous studies of proteins $[5,10,11,19,20]$. The vector dimensional of physicochemical property feature is 132 .

2.2.3. Evolutionary Information (EI). Position-specific scoring matrix (PSSM) which represents evolutionary information of amino acid sequences was used mostly in the prediction of DNA-binding residues [24-29] and plays an important role in distinguishing DNA-binding residues from nonbinding residues in those researches. Therefore, we considered to apply PSSM to identify DNA-binding protein in this research. PSSM scores are generated by PSI-BLAST [30] to search against the nonredundant dataset of amino acid sequences in NCBI, and 20 values are obtained for each sequence position. If the protein has $N$ amino acids, the feature vector of PSSM is $20 * N$. However, different proteins may have different number of amino acids. PSSM could not directly be used as a feature in the prediction work based on machine learning method. In order to convert variable feature vector into fixed vector, we improved PSSM in following step.

First, we normalized the values of PSSM using formula as follows:

$$
p(x)=\frac{1}{1+\exp (-x)}
$$

Second, we pooled all rows which belong to the same amino acid in this PSSM and together to form a new matrix. Then we obtained 20 new matrices with the size $N a * 20$, where $\mathrm{Na}$ is the number of amino acids of type $a$.

Third, we converted each new matrix to a vector. We added all values in each column in new matrices. For each new matrix, we produced a 20 -dimensional vector. Then we obtained $20 * 20=400$ dimension vector which represents feature of evolutionary information in this work.

2.3. Algorithms for Classification. Five machine learning algorithms were used in our study to select a bestperformance algorithm to identify DNA-binding proteins: support vector machine-sequential minimal optimization (SVM-SMO) [31], simple logistic regression [32], random forest [33], naive Bayes [34], and decision tree [35].

Support vector machine (SVM) [36] is a supervise machine learning algorithm and widely applied in classification researches. The principle of SVM is to find a hyperplane as a segmentation of two classes to minimize the classified error. Sequential minimal optimization (SMO) is an algorithm for training support vector machine to efficiently solve the optimization problem. Simple logistic regression is a statistical model suitable for probabilistic binary classification. Random forest is a classification algorithm that uses an ensemble of tree-structured classifiers. Naive Bayes classifier technique is a simple probabilistic classifier based on applying Bayesian theorem independence assumptions and 
is particularly suited when the dimensionality of the inputs is high. Decision tree classifier generates tree-like graph or model of decisions for classification. It is developed using the classification and regression trees method.

These machine learning algorithms are implemented by WEKA software (http://www.cs.waikato.ac.nz/ ml/weka/) [37] which provides a collection of machine learning algorithms for data mining tasks.

2.4. Model Evaluation Procedure. To obtain a reliable result with low mean square error, $k$-fold cross validation was always used in empirical works [7, 8]. In this study, 5-fold cross validation method was used to access the performance of each classifier on the main dataset. The main dataset was randomly divided into 5 equal parts. Each run of cross validation is comprised of one part as the independent test dataset and remaining 4 parts as the training dataset. For each classification, performance evaluations of 5 repetitions of 5fold cross validation were averaged and calculated.

The following performance evaluations were calculated: accuracy, sensitivity, specificity, and Matthew correlation coefficient (MCC) [4]. Equations of the performance evaluations were represented as follows:

$$
\begin{gathered}
\text { Accuary }=\frac{\mathrm{TP}+\mathrm{TN}}{\mathrm{TP}+\mathrm{FP}+\mathrm{TN}+\mathrm{FN}}, \\
\text { Sensitivity }=\frac{\mathrm{TP}}{\mathrm{TP}+\mathrm{FN}}, \\
\text { Specificity }=\frac{\mathrm{TN}}{\mathrm{TN}+\mathrm{FP}}, \\
\mathrm{MCC}=\frac{\mathrm{TP} \times \mathrm{TN}-\mathrm{FP} \times \mathrm{FN}}{\sqrt{(\mathrm{TP}+\mathrm{FP})(\mathrm{TN}+\mathrm{FN})(\mathrm{TP}+\mathrm{FN})(\mathrm{TN}+\mathrm{FP})}} .
\end{gathered}
$$

ROC curve is widely accepted and effective method to compare the overall prediction performance of different classifier method. An ROC curve is constructed by plotting the sensitivity versus 1-specificity for varying cutoff values. The area under the curve (AUC) is the evaluation criteria for the classifier. Therefore, we used AUC values to compare our work with previous studies $[15,16]$.

\section{Result and Discussion}

3.1. Performance of Various Machine Learning Algorithms. The performance is shown in Table 2 for predicting DNAbinding proteins based on support vector machine-sequential minimal optimization (SVM-SMO), simple logistic regression, random forest, naive Bayes, and decision tree with 5fold cross validation on main dataset. As shown in Table 2, the results demonstrate that SVM-SMO classifier achieves the best performance. The combination of all features achieves the best performance with accuracy, sensitivity, specificity, Matthew correlation coefficient, and AUC equal to $89.6 \%$, $88.4 \%, 90.8 \%, 0.67$, and 0.90 , respectively. The performance of simple logistic regression classifier is slightly worse than that of SVM-SMO classifier but much better than other three classifiers with $88.3 \%$ accuracy, $86.7 \%$ sensitivity, $90.2 \%$ specificity, 0.66 Matthew correlation coefficient, and 0.88 AUC. Naive Bayes classifier achieves the worst performance which predicted DNA-binding proteins at $84.3 \%$ accuracy with Matthew's correlation coefficient of 0.59 , and with a sensitivity of $82.6 \%$ and a specificity of $86.0 \%$. The performance of random forest classifier is better than decision tree classifier. Considering the performance, we chose SVM-SMO classifier to identify DNA-binding proteins in our research.

3.2. Importance of Novel Attributes: Binding Propensity and Nonbinding Propensity. The novel attributes: binding propensity (BP) and nonbinding propensity (NBP), were firstly proposed in this research. Those two features were constructed by prediction results from our earlier developed prediction model DNABR which has excellent prediction performance in DNA-binding residues. In order to know the importance of those two features, $\mathrm{BP}$ and NB were combined with evolutionary information feature (EI) and physicochemical property feature (PP) to construct DNA-binding proteins prediction model using SVM-SMO algorithm, respectively. Seen from Table 2, when BP and NBP were combined with $\mathrm{PP}$, the value of accuracy significantly increased and achieved 85.6\%. The similar result were appeared when BP and NBP were combined with EI, and the value of accuracy increased considerably to $87.3 \%$ with MCC 0.66 . Those results which were evaluated by 5 -fold cross validation proved that BP and NBP play a significant role in distinguishing binding proteins from nonbinding proteins.

Figures 1(a) and 1(b) present that binding and nonbinding proteins show contrasting behavior in terms of two components of BP feature. Figures 2(a) and 2(b) also show significant difference between binding and nonbinding proteins in terms of two components of NBP feature. We also calculated the $P$ values of two BP components and two NBP components to measure the ability to separate the binding proteins from the nonbinding ones. Each of them was less than 0.00005 . These results also proved that BP and NBP play a vital role in achieving excellent performance in our study.

The character of DNA-binding proteins and nonbinding proteins can illuminate the importance of BP and NBP features. (1) It is obvious that each DNA-binding protein has several residues which bind to DNA. DNA-binding residues should be much more in DNA-binding proteins in comparison to nonbinding proteins. The structural distribution of DNA-binding residues also has some regular pattern, such as DNA-binding residues which tend to gather together spatially on the surface of DNA-binding protein [38]. Two components of BP feature revealed the character of DNA-binding proteins for sequence level and spatial level, respectively. (2) On the contrary, proportion of nonbinding residues should be much higher for nonbinding proteins in comparison to DNA-binding proteins. Hence it is rational to propose NBP feature. Therefore, we predicted DNAbinding residues and nonbinding residues in a query protein by DNABR model which achieved the best performance in comparison to previous similar works. It was observed that using information of binding and nonbinding residues 
TABLE 2: The performance of different kinds of feature descriptors with various machine learning algorithms based on main dataset using 5 -fold cross-validation.

\begin{tabular}{|c|c|c|c|c|c|c|}
\hline \multirow{2}{*}{ Machine learning algorithm } & \multicolumn{6}{|c|}{ Feature descriptor } \\
\hline & $\mathrm{PP}$ & EI & $\mathrm{BP}+\mathrm{NBP}+\mathrm{PP}$ & $\mathrm{BP}+\mathrm{NBP}+\mathrm{EI}$ & $\mathrm{PP}+\mathrm{EI}$ & $\mathrm{BP}+\mathrm{NBP}+\mathrm{PP}+\mathrm{EI}$ \\
\hline & \multicolumn{6}{|c|}{ Accuracy (\%) } \\
\hline SVM-SMO & 83.2 & 85.7 & 85.6 & 87.3 & 87.3 & 89.6 \\
\hline Simple logistic regression & 81.8 & 84.2 & 84.2 & 87.0 & 85.7 & 88.3 \\
\hline Random forest & 81.3 & 84.3 & 83.5 & 86.7 & 85.9 & 88.1 \\
\hline Naive bayes & 78.6 & 77.2 & 82.8 & 82.3 & 82.6 & 84.3 \\
\hline \multirow[t]{2}{*}{ Decision tree } & 80.2 & 82.5 & 82.6 & 84.4 & 84.1 & 86.2 \\
\hline & \multicolumn{6}{|c|}{ Sensitivity (\%) } \\
\hline SVM-SMO & 82.4 & 84.9 & 84.4 & 86.5 & 85.8 & 88.4 \\
\hline Simple logistic regression & 80.7 & 83.1 & 82.3 & 84.4 & 85.6 & 86.7 \\
\hline Random forest & 81.1 & 83.6 & 82.8 & 86.0 & 85.3 & 86.2 \\
\hline Naive bayes & 76.9 & 76.1 & 79.4 & 80.8 & 81.1 & 82.6 \\
\hline \multirow[t]{2}{*}{ Decision tree } & 78.6 & 80.4 & 81.7 & 82.7 & 82.5 & 84.7 \\
\hline & \multicolumn{6}{|c|}{ Specificity (\%) } \\
\hline SVM-SMO & 84.6 & 86.3 & 86.7 & 88.2 & 88.6 & 90.8 \\
\hline Simple logistic regression & 82.9 & 85.5 & 86.0 & 88.8 & 85.9 & 90.2 \\
\hline Random forest & 81.6 & 85.2 & 84.1 & 87.5 & 86.3 & 90.0 \\
\hline Naive bayes & 80.2 & 78.5 & 85.6 & 83.8 & 84.7 & 86.0 \\
\hline \multirow[t]{2}{*}{ Decision tree } & 81.8 & 84.7 & 83.5 & 86.2 & 85.7 & 87.7 \\
\hline & \multicolumn{6}{|c|}{ Matthew correlation coefficient } \\
\hline SVM-SMO & 0.55 & 0.58 & 0.62 & 0.66 & 0.66 & 0.67 \\
\hline Simple logistic regression & 0.56 & 0.55 & 0.64 & 0.62 & 0.64 & 0.66 \\
\hline Random forest & 0.55 & 0.56 & 0.60 & 0.62 & 0.63 & 0.66 \\
\hline Naive bayes & 0.52 & 0.49 & 0.56 & 0.53 & 0.54 & 0.59 \\
\hline \multirow[t]{2}{*}{ Decision tree } & 0.53 & 0.55 & 0.61 & 0.63 & 0.62 & 0.64 \\
\hline & \multicolumn{6}{|c|}{ AUC } \\
\hline SVM-SMO & 0.83 & 0.86 & 0.86 & 0.88 & 0.87 & 0.90 \\
\hline Simple logistic regression & 0.83 & 0.84 & 0.85 & 0.86 & 0.85 & 0.88 \\
\hline Random forest & 0.81 & 0.84 & 0.84 & 0.86 & 0.85 & 0.87 \\
\hline Naive bayes & 0.78 & 0.76 & 0.80 & 0.79 & 0.80 & 0.82 \\
\hline Decision tree & 0.80 & 0.82 & 0.83 & 0.84 & 0.84 & 0.86 \\
\hline
\end{tabular}

BP: binding propensity feature; NBP: nonbinding propensity feature; PP: physicochemical property feature; EI: evolutionary information feature.

worked well as we expected. BP and NBP can successfully discriminate between DNA-binding proteins and nonbinding proteins.

3.3. Performance Comparison with Other Methods. iDNAProt (http://www.jci-bioinfo.cn/iDNA-Prot) [16] predicts a query protein as a DNA-binding protein or a nonDNA- binding protein only based on its amino acid sequence information. The iDNA-Prot was constructed by incorporating the features into the general form of pseudo amino acid composition that was extracted from protein sequences via the "grey model" and by adopting the random forest model. The overall success rate by iDNA-Prot was 83.96\%. Kumar et al. proposed a random forest method,
DNA-Prot, to identify DNA-binding proteins from protein sequence [15]. DNA-Prot was proposed to encode each protein sequence with 116 features by incorporating various physicochemical properties of amino acids. Using the dataset in research [15] DNA-Prot could identify DNAbinding proteins from non-DNA-binding proteins with more than $80 \%$ accuracy. As mentioned in Section 2, to evaluate the performance of our method against the stateof-the-art algorithms, that is, iDNA-Prot and DNA-Prot, an independent test dataset $\mathrm{TeD} \_2664$ was obtained by selecting 1332 DNA-binding proteins and 1332 nonbinding proteins randomly from "Positive" dataset and "Negative subset" dataset, respectively. The iDNA-Prot prediction results of 2664 proteins in TeD_2664 dataset were obtained by webserver (http://www.jci-bioinfo.cn/iDNA-Prot). TeD_2664 


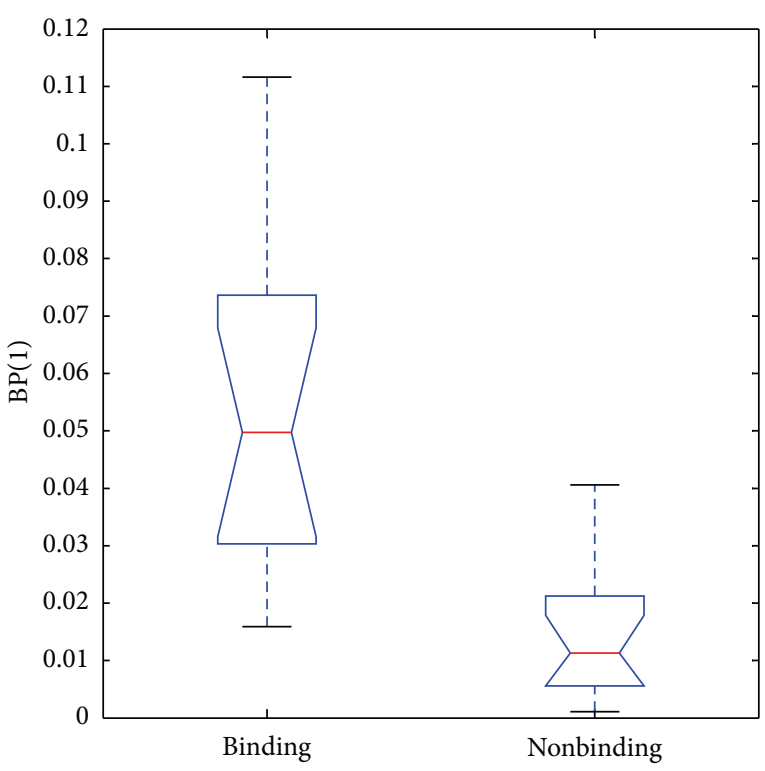

(a)

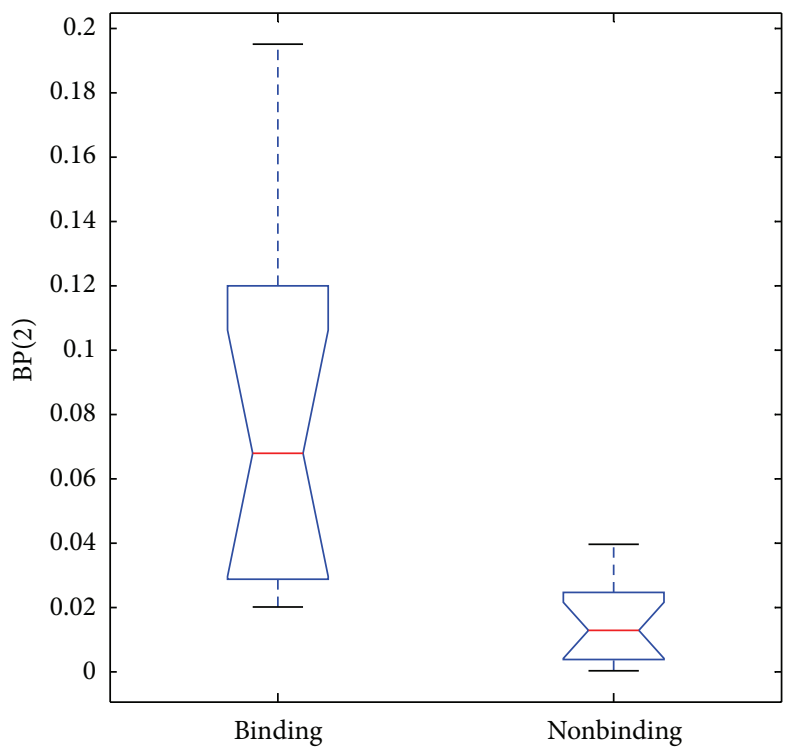

(b)

FIGURE 1: Box plots of the two components of BP feature for binding and nonbinding proteins. (a) BP(1); (b) BP(2).

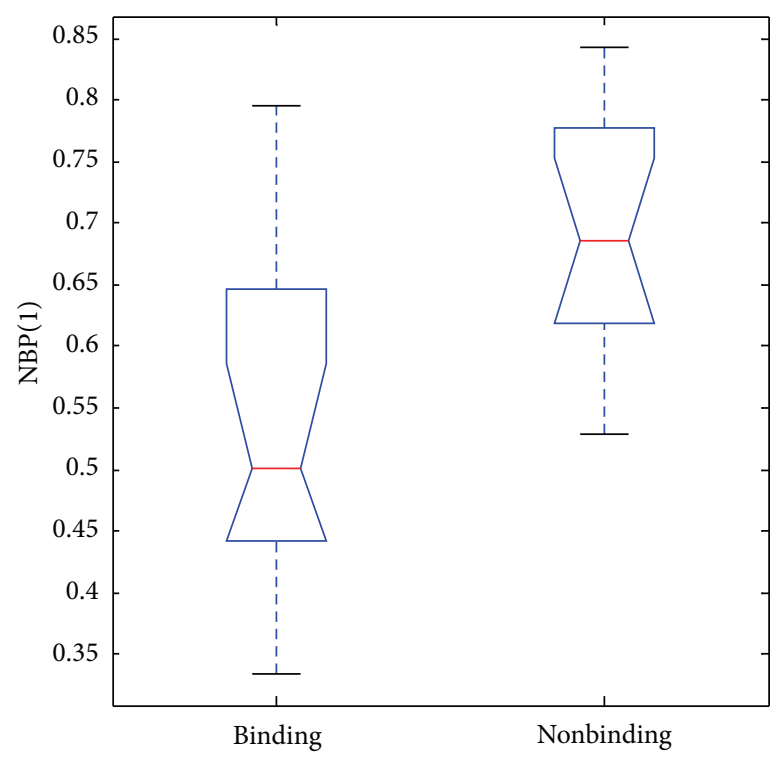

(a)

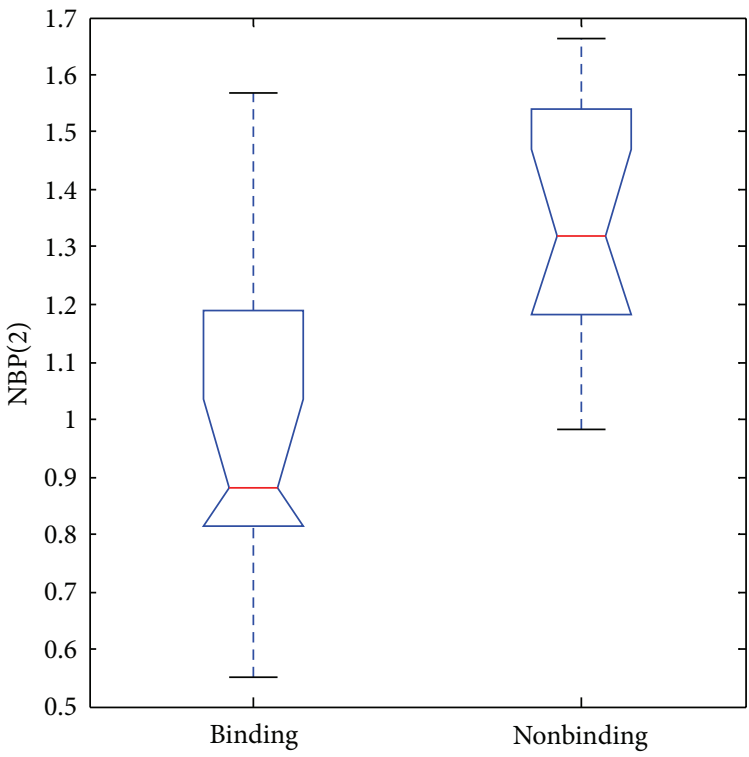

(b)

FIGURE 2: Box plots of the two components of NBP feature for binding and nonbinding proteins. (a) NBP(1); (b) NBP(2).

dataset used to test DNA-Prot and the prediction results can be obtained from its standalone version (http://www3.ntu .edu.sg/home/EPNSugan/index files/dnaprot.htm). Here we also trained another SVM-SMO model on training dataset TrD_10642 using the same strategy as original SVM-SMO model with all features and the model used to identify DNA-binding proteins in the TeD_2664 dataset. As shown in Figure 3 , the accuracy is $74.88 \%, 54.06 \%$, and $47.30 \%$ for our SVM-SMO model, iDNA-Prot, and DNA-Prot, respectively. The SVM-SMO model attained $72.22 \%$ sensitivity, $77.55 \%$ specificity, and 0.4981 MCC. The results show that the SVM-SMO model achieves the best performance.

\section{Conclusions}

In this paper, we present a novel approach based on support vector machine-sequential minimal optimization (SVMSMO) and a hybrid feature for the prediction of DNA-binding proteins using only the primary sequence of a protein. Two novel attributes, denoting DNA-binding propensity and nonbinding propensity, were constructed by DNA-binding residues information and nonbinding residues information. The results prove that these two attributes markedly improve the prediction performance. The SVM-SMO model with the hybrid feature that includes two novel attributes, evolutionary 


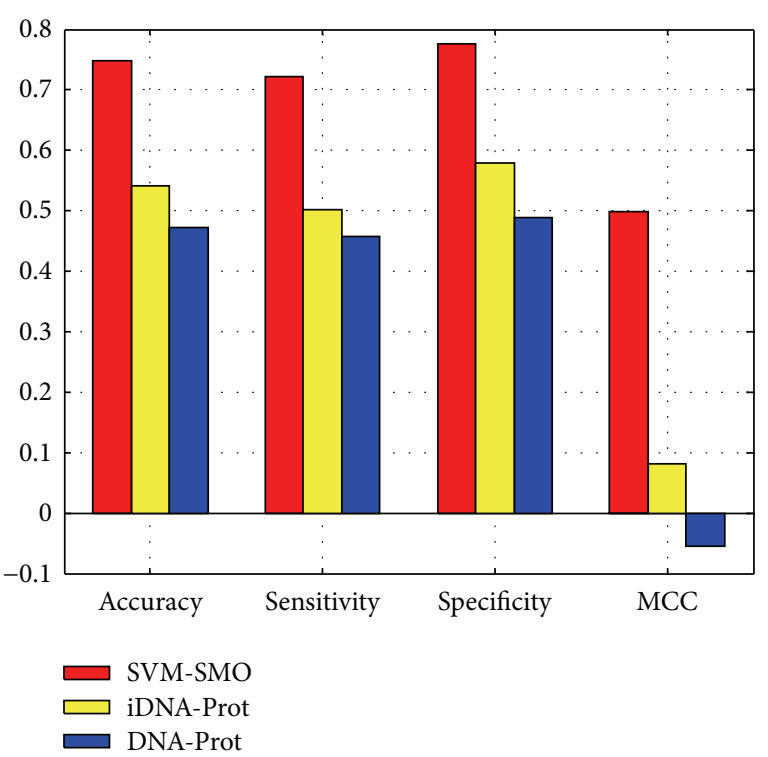

Figure 3: Three classifiers were tested on the same testing dataset TeD_2264. The predictors have the following accuracy value: our SVM-SMO 74.88\%, iDNA-Prot 54.06\%, and DNA-Prot 47.30\%; sensitivity: our SVM-SMO 72.22\%, iDNA-Prot 50.22\%, and DNA-Prot 45.72\%; specificity: our SVM-SMO 77.55\%, iDNA-Prot 57.88\%, and DNA-Prot 48.87\%; MCC: our SVM-SMO 0.4981, iDNA-Prot0.0814, and DNA-Prot -0.0541 .

information feature, and physicochemical property feature has a prediction accuracy of $89.6 \%$ with MCC of 0.67 . We believe that our SVM-SMO method is currently the most effective method for predicting DNA-binding proteins using only sequence information.

\section{Conflict of Interests}

The authors confirm that there is no conflict of interests in relation to this work.

\section{Acknowledgments}

This work was supported by the National Natural Science Foundation of China (no. 61305072 and no. 61203289), Natural Science Foundation of the Jiangsu Higher Education Institutions of China (no. 12KJD520006 and no. 12KJB520010), and the Open Research Fund of State Key Laboratory of Bioelectronics, Southeast University (no. BK212001).

\section{References}

[1] C. J. Drummond, G. J. Finlay, L. Broome, E. S. Marshall, E. Richardson, and B. C. Baguley, "Action of SN 28049, a new DNA binding topoisomerase II-directed antitumour drug: comparison with doxorubicin and etoposide," Investigational New Drugs, vol. 29, no. 5, pp. 1102-1110, 2011.

[2] H. Gao and K. Dahlman-Wright, "From DNA binding to metabolic control: integration of-omics data reveals drug targets for prostate cancer," EMBO Journal, vol. 30, no. 13, pp. 2516-2517, 2011.
[3] G. Y. Park, J. J. Wilson, Y. Song, and S. J. Lippard, "Phenanthriplatin, a monofunctional DNA-binding platinum anticancer drug candidate with unusual potency and cellular activity profile," Proceedings of the National Academy of Sciences of the United States of America, vol. 109, no. 30, pp. 11987-11992, 2012.

[4] H. Zhao, Y. Yang, and Y. Zhou, "Structure-based prediction of DNA-binding proteins by structural alignment and a volumefraction corrected DFIRE-based energy function," Bioinformatics, vol. 26, no. 15, Article ID btq295, pp. 1857-1863, 2010.

[5] S. Ahmad, M. M. Gromiha, and A. Sarai, "Analysis and prediction of DNA-binding proteins and their binding residues based on composition, sequence and structural information," Bioinformatics, vol. 20, no. 4, pp. 477-486, 2004.

[6] W. A. McLaughlin, D. W. Kulp, J. de la Cruz, X. Lu, C. L. Lawson, and H. M. Berman, "A structure-based method for identifying DNA-binding proteins and their sites of DNA-interaction," Journal of Structural and Functional Genomics, vol. 5, no. 4, pp. 255-265, 2005.

[7] G. Nimrod, A. Szilágyi, C. Leslie, and N. Ben-Tal, "Identification of DNA-binding proteins using structural, electrostatic and evolutionary features," Journal of Molecular Biology, vol. 387, no. 4, pp. 1040-1053, 2009.

[8] A. Szaboova, O. Kuzelka, F. Zelezny, and J. Tolar, "Prediction of DNA-binding proteins from relational features," Proteome Science, vol. 10, no. 1, article 66, 2012.

[9] N. Bhardwaj, R. E. Langlois, G. Zhao, and H. Lu, "Kernelbased machine learning protocol for predicting DNA-binding proteins," Nucleic Acids Research, vol. 33, no. 20, pp. 6486-6493, 2005.

[10] Y. D. Cai and S. L. Lin, "Support vector machines for predicting rRNA-, RNA-, and DNA-binding proteins from amino acid sequence," Biochimica et Biophysica Acta, vol. 1648, no. 1-2, pp. 127-133, 2003.

[11] X. Yu, J. Cao, Y. Cai, T. Shi, and Y. Li, "Predicting rRNA-, RNA-, and DNA-binding proteins from primary structure with support vector machines," Journal of Theoretical Biology, vol. 240, no. 2, pp. 175-184, 2006.

[12] M. Kumar, M. M. Gromiha, and G. P. S. Raghava, "Identification of DNA-binding proteins using support vector machines and evolutionary profiles," BMC Bioinformatics, vol. 8, article 463, 2007.

[13] X. Shao, Y. Tian, L. Wu, Y. Wang, L. Jing, and N. Deng, "Predicting DNA- and RNA-binding proteins from sequences with kernel methods," Journal of Theoretical Biology, vol. 258, no. 2, pp. 289-293, 2009.

[14] A. K. Patel, S. Patel, and P. K. Naik, "Binary classification of uncharacterized proteins into DNA binding/non-DNA binding proteins from sequence derived features using ANN," Digest Journal of Nanomaterials and Biostructures, vol. 4, no. 4, pp. 775-782, 2009.

[15] K. K. Kumar, G. Pugalenthi, and P. N. Suganthan, "DNA-prot: identification of DNA binding proteins from protein sequence information using random forest," Journal of Biomolecular Structure and Dynamics, vol. 26, no. 6, pp. 679-686, 2009.

[16] W. Z. Lin, J. A. Fang, X. Xiao, and K. C. Chou, "iDNA-prot: identification of DNA binding proteins using random forest with grey model," PLoS ONE, vol. 6, no. 9, Article ID e24756, 2011.

[17] X. Ma, J. Guo, H. D. Liu, J. M. Xie, and X. Sun, "Sequencebased prediction of DNA-binding residues in proteins with conservation and correlation information," IEEE Transactions 
on Computational Biology and Bioinformatics, vol. 9, no. 6, pp. 1766-1775, 2012.

[18] T. U. Consortium, "Reorganizing the protein space at the universal protein resource (UniProt)," Nucleic Acids Research, vol. 40, no. 1, pp. D71-D75, 2012.

[19] S. Ahmad and A. Sarai, "Moment-based prediction of DNAbinding proteins," Journal of Molecular Biology, vol. 341, no. 1, pp. 65-71, 2004.

[20] C. R. Peng, L. Liu, B. Niu et al., "Prediction of RNA-binding proteins by voting systems," Journal of Biomedicine and Biotechnology, vol. 2011, Article ID 506205, 8 pages, 2011.

[21] J. R. Bock and D. A. Gough, "Predicting protein-protein interactions from primary structure," Bioinformatics, vol. 17 , no. 5 , pp. 455-460, 2001.

[22] C. H. Q. Ding and I. Dubchak, "Multi-class protein fold recognition using support vector machines and neural networks," Bioinformatics, vol. 17, no. 4, pp. 349-358, 2001.

[23] C. Z. Cai, L. Y. Han, Z. L. Ji, X. Chen, and Y. Z. Chen, "SVMProt: web-based support vector machine software for functional classification of a protein from its primary sequence," Nucleic Acids Research, vol. 31, no. 13, pp. 3692-3697, 2003.

[24] S. Ahmad and A. Sarai, "PSSM-based prediction of DNA binding sites in proteins," BMC Bioinformatics, vol. 6, article 33, 2005.

[25] S. Y. Ho, F. C. Yu, C. Y. Chang, and H. L. Huang, "Design of accurate predictors for DNA-binding sites in proteins using hybrid SVM-PSSM method," BioSystems, vol. 90, no. 1, pp. 234241, 2007.

[26] L. Wang, M. Q. Yang, and J. Y. Yang, "Prediction of DNAbinding residues from protein sequence information using random forests," BMC Genomics, vol. 10, supplement 1, article S1, 2009.

[27] J. Wu, H. Liu, X. Duan et al., "Prediction of DNA-binding residues in proteins from amino acid sequences using a random forest model with a hybrid feature," Bioinformatics, vol. 25, no. 1, pp. 30-35, 2009.

[28] X. Ma, J. Wu, H. Liu, X. Yang, J. Xie, and X. Sun, “A SVM-based approach for predicting DNA-binding residues in proteins from amino acid sequences," in Proceedings of the International Joint Conference on Bioinformatics, Systems Biology and Intelligent Computing (IJCBS '09), pp. 225-229, Shanghai, China, August 2009.

[29] L. Wang, C. Huang, M. Q. Yang, and J. Y. Yang, "BindN+ for accurate prediction of DNA and RNA-binding residues from protein sequence features," BMC Systems Biology, vol. 4, supplement 1, article S3, 2010.

[30] S. F. Altschul, T. L. Madden, A. A. Schäffer et al., "Gapped BLAST and PSI-BLAST: a new generation of protein database search programs," Nucleic Acids Research, vol. 25, no. 17, pp. 3389-3402, 1997.

[31] J. C. Platt, "Sequential minimal optimization: a fast algorithm for training support vector machine," Microsoft Research, Technical Report MSR-TR-98-14, 1998.

[32] N. Landwehr, M. Hall, and E. Frank, "Logistic model trees," Machine Learning, vol. 59, no. 1-2, pp. 161-205, 2005.

[33] L. Breiman, "Random forests," Machine Learning, vol. 45, no. 1, pp. 5-32, 2001.

[34] P. Domingos and M. Pazzani, "On the optimality of the simple Bayesian classifier under zero-one loss," Machine Learning, vol. 29, no. 2-3, pp. 103-130, 1997.
[35] L. Rokach and O. Maimon, Data Mining with Decision Trees: Theory and Applications, World Scientific, River Edge, NJ, USA, 2008.

[36] V. N. Vapnik, Statistical Learning Theory, John Wiley \& Sons, New York, NY, USA, 1998.

[37] M. Hall, E. Frank, G. Holmes, B. Pfahringer, P. Reutemann, and I. H. Witten, "The WEKA data mining software: an update," SIGKDD Explorations, vol. 11, no. 1, pp. 10-18, 2009.

[38] S. Campagne, V. Gervais, and A. Milon, "Nuclear magnetic resonance analysis of protein-DNA interactions," Journal of the Royal Society Interface, vol. 8, no. 61, pp. 1065-1078, 2011. 


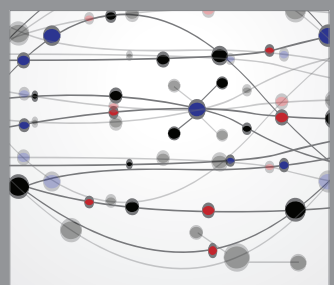

The Scientific World Journal
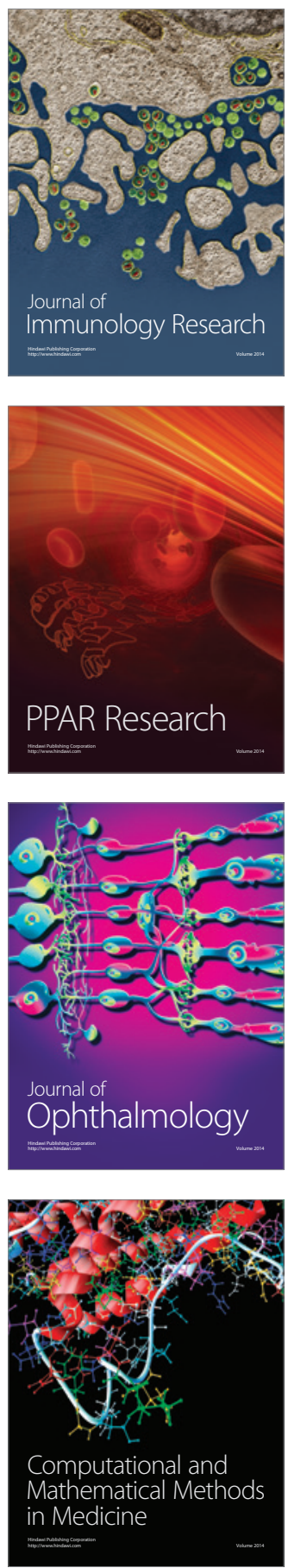

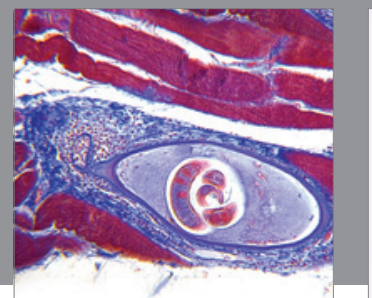

Gastroenterology

Research and Practice
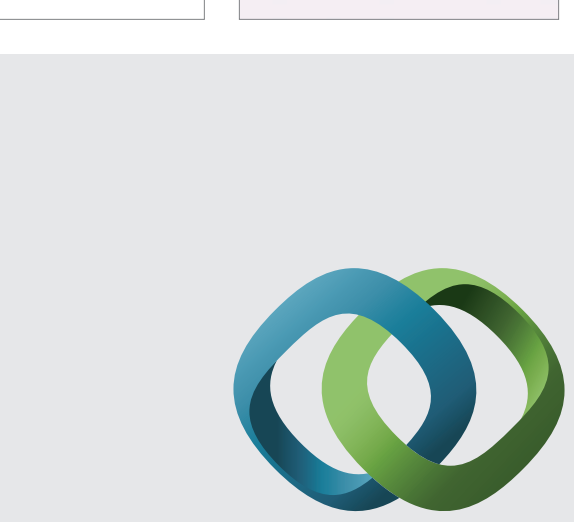

\section{Hindawi}

Submit your manuscripts at

http://www.hindawi.com
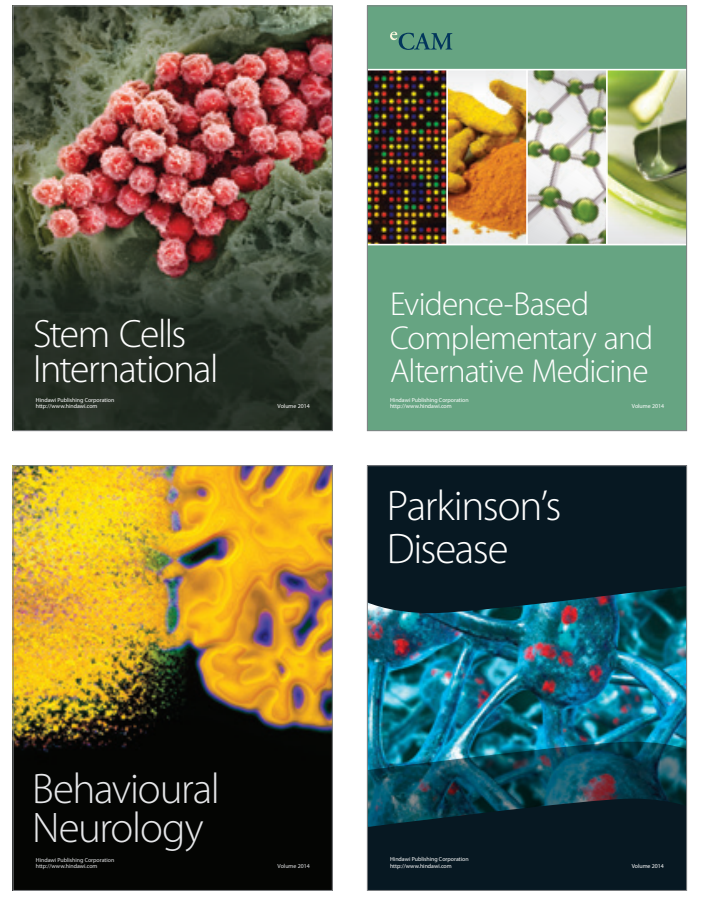
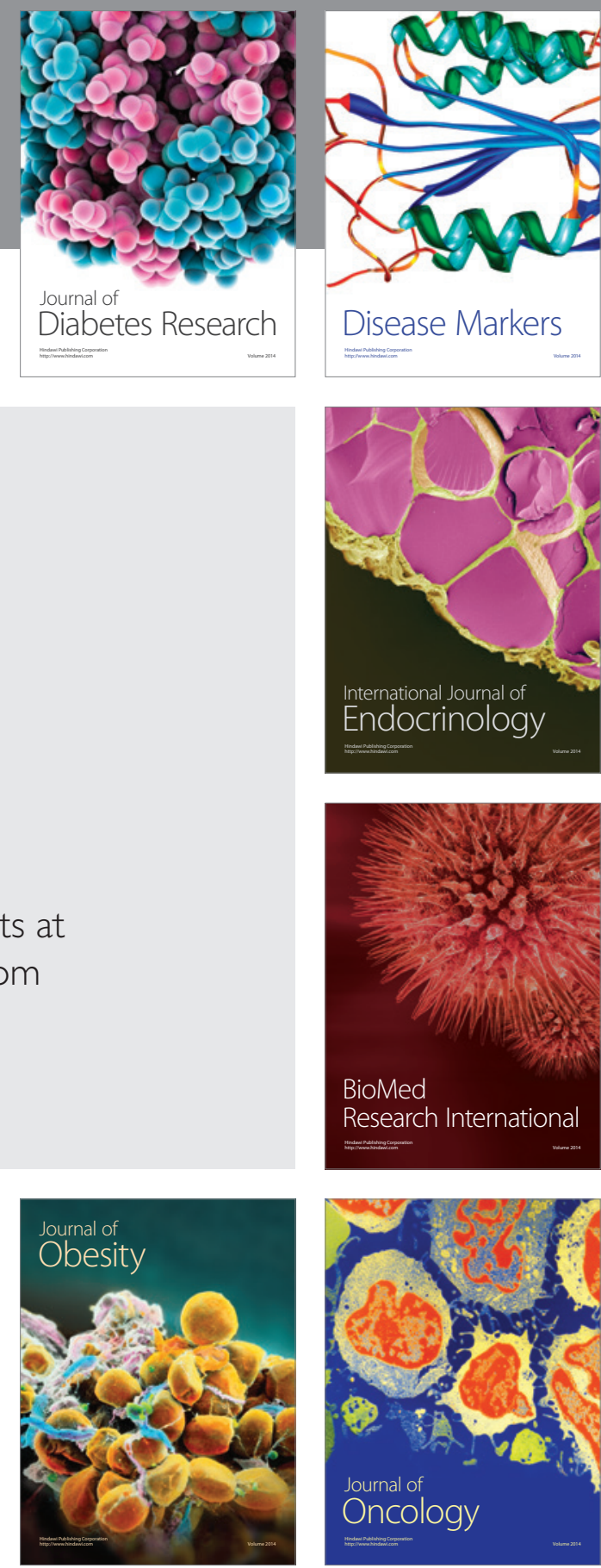

Disease Markers
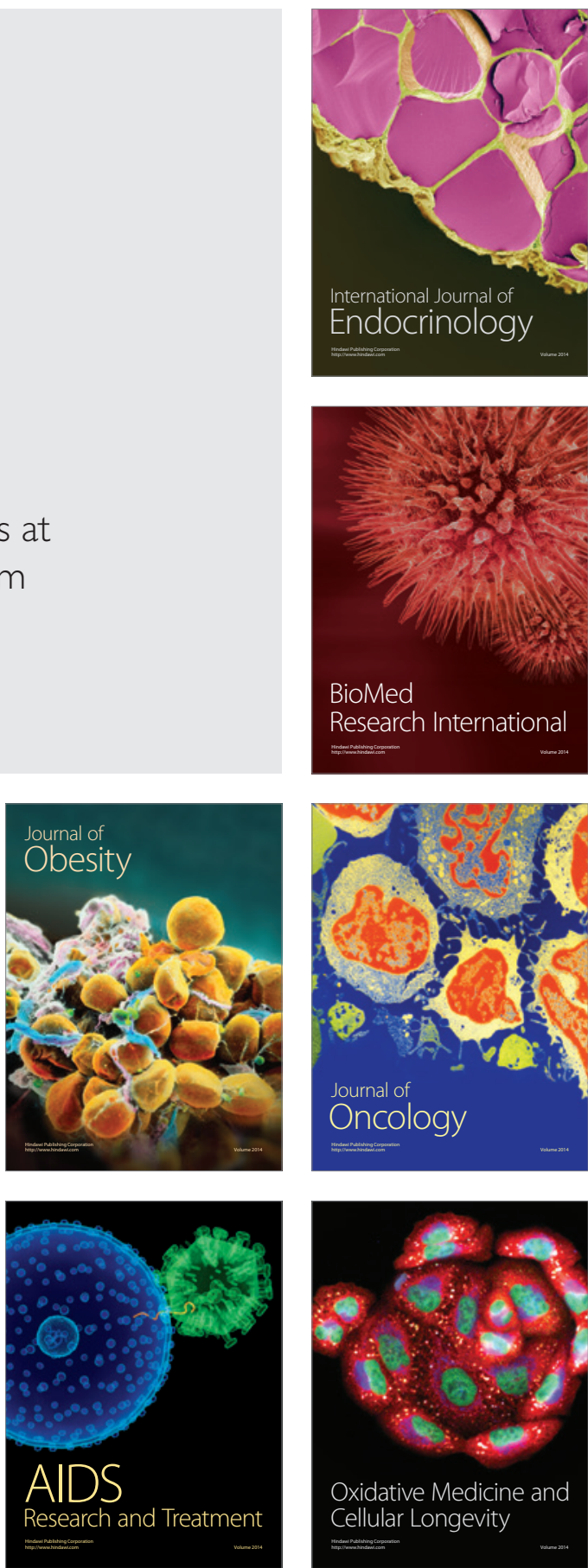